\title{
Investigating the Significance of Multiple Scattering in Ultrasound Contrast Agent Particle Populations
}

\author{
Eleanor Stride and Nader Saffari
}

\begin{abstract}
The majority of the existing models describing the behavior of microbubble ultrasound contrast agents consider single, isolated microbubbles suspended in infinite media. The behavior of a microbubble population is predicted by summing the results for single microbubbles and ignoring multiple scattering effects. The aim of this investigation is to determine the significance of multiple scattering in microbubble populations and establish whether an alternative approach is required. In the first part of the work, linear models are derived to identify approximately the conditions under which multiple scattering may be expected. A nonlinear model for sound propagation in a microbubble suspension then is developed and used to examine multiple scattering at higher insonation pressures. Broadband attenuation measurements are described for two different types of microbubble suspension (albumin encapsulated octofluropropane and copolymer encapsulated isobutane) to ascertain whether or not multiple scattering may be observed experimentally. The results from the simulation work indicate that multiple scattering effects would be discernible at moderate concentrations $\left(10^{6}\right.$ microbubbles/ml) such as may be present in vivo. The effect upon attenuation in the suspension would be pronounced, however, only if the population contained a sufficient proportion of relatively large $(>4 \mu \mathrm{m}$ radius) microbubbles excited at their resonance frequency. This also is found to be the case experimentally. These findings may have important implications for the characterization of ultrasound contrast agents and their use in quantitative diagnostic techniques.
\end{abstract}

\section{INTRODUCTION}

$\mathrm{U}$ LTRASOUND contrast agents consisting of gas microbubbles coated with a surfactant or polymer shell have been in clinical use for a number of years. Their benefits have been demonstrated in a range of imaging applications and, more recently, their use in therapeutic applications such as targeted drug delivery has become an active area of research [1]. However, their behavior in vivo is by no means fully understood, owing to the complexity of the modeling involved. The aim of this investigation is to examine the problem of multiple scattering in contrast agent suspensions.

The majority of the existing models for the behavior of ultrasound contrast agents consider single, isolated microbubbles suspended in infinite media (e.g., [2], [3]). In

Manuscript received November 1, 2004; accepted May 27, 2005.

The authors are with the Department of Mechanical Engineering, University College London, London WC1E 7JE, UK (e-mail: e_stride@meng.ucl.ac.uk). order to predict the behavior of a microbubble population, there are assumed to be no interactions between individual microbubbles, and the results for single microbubbles are simply summed after being weighted according to the size distribution in the suspension. It is known, however, that the effectiveness of microbubbles is due to the fact that they are strong backscatterers of ultrasound, much stronger than red blood cells. Therefore, it would seem reasonable to suppose that, at certain concentrations and frequencies, there will be multiple scattering between microbubbles that will affect the overall response of the population. This supposition is supported by a number of experimental studies (e.g., [4], [5]) in which discrepancies between measurements and the predictions from single microbubble theories have been identified. If contrast agent behavior is to be fully understood, it is essential to determine the conditions under which multiple scattering should be taken into account.

The specific objectives of this investigation are:

- To derive linear multiple scattering models for microbubble suspensions to identify approximately the conditions under which the effects of multiple scattering are likely to be significant.

- To develop a nonlinear multiple scattering model to investigate the phenomenon in microbubble suspensions at higher insonation pressures.

- To test the validity of the theoretical results experimentally.

\section{THEORY}

\section{A. Coated Microbubble Dynamics}

The equation of motion for a spherical coated microbubble in the form given by Church [3] may be written as:

$$
\begin{aligned}
& R_{1} \ddot{R}_{1}\left(1+\left(\frac{\rho_{L}-\rho_{s}}{\rho_{s}}\right) \frac{R_{1}}{R_{2}}\right) \\
&+\dot{R}_{1}^{2}\left(\frac{3}{2}+\left(\frac{\rho_{L}-\rho_{s}}{\rho_{s}}\right)\left(\frac{4 R_{2}^{3}-R_{1}^{3}}{2 R_{2}^{3}}\right) \frac{R_{1}}{R_{2}}\right) \\
&=\frac{1}{\rho_{s}}\left(\begin{array}{l}
p_{o}\left(\frac{R_{o 1}}{R_{1}}\right)^{3 \kappa}-p_{\infty}(t)-\frac{2 \sigma_{1}}{R_{1}}-\frac{2 \sigma_{2}}{R_{2}} \\
-4 \frac{\dot{R}_{1}}{R_{1}}\left(\frac{V_{s} \mu_{s}-R_{1}^{3} \mu_{L}}{R_{2}^{3}}\right)-\frac{4 V_{s} G_{s}}{R_{2}^{3}}\left(1-\frac{R_{1 e}}{R_{1}}\right)-b_{\mathrm{rad}}
\end{array}\right),(1)
\end{aligned}
$$


TABLE I

List of Symbols.

\begin{tabular}{|c|c|c|c|}
\hline$a$ & attenuation coefficient & $\dot{R}_{1}$ & radial velocity of inner microbubble shell surface \\
\hline$A_{1}$ & signal amplitude in water & $\ddot{R}_{1}$ & radial acceleration of inner microbubble shell surface \\
\hline$A_{2}$ & signal amplitude in microbubble suspension & $R_{1 e}$ & equilibrium inner microbubble radius \\
\hline$b_{\text {rad }}$ & acoustic radiation damping factor & $R_{2}$ & outer microbubble radius \\
\hline$c_{e f f}$ & acoustic velocity in the effective medium & $R_{o 1}$ & initial inner microbubble radius \\
\hline$c_{L}$ & acoustic velocity in the surrounding fluid & $R_{o 2}$ & initial outer microbubble radius \\
\hline$d$ & propagation distance & $s_{\text {ext }}$ & extinction cross section \\
\hline$f$ & scattering function & $t$ & time \\
\hline$F$ & scattering function for higher order scattering & $\mathbf{u}$ & radial velocity \\
\hline$G_{s}$ & shell stiffness parameter & $V s$ & microbubble shell volume factor \\
\hline$h\left(R_{o 2}, x\right)$ & microbubble size distribution function & $x$ & linearization factor \\
\hline$I_{i}$ & incident intensity & $y$ & location in space \\
\hline$i$ & space discretization index & $z$ & microbubble shell factor \\
\hline$j$ & time discretization index & $\alpha$ & microbubble shell factor \\
\hline$K_{\text {eff }}$ & wave number in the effective medium & $\beta$ & microbubble volume fraction \\
\hline$k_{L}$ & wave number in the surrounding fluid & $\delta_{d}$ & net damping factor \\
\hline$M$ & number of time steps & $\delta_{\text {rad }}$ & acoustic radiation damping factor \\
\hline$m$ & pulse shape parameter & $\phi$ & phase angle \\
\hline$N$ & number of steps in space & $\kappa$ & polytropic constant \\
\hline$n$ & microbubble concentration & $\mu_{L}$ & viscosity of the surrounding fluid \\
\hline$p$ & pressure & $\mu_{S}$ & viscocity of the encapsulating shell \\
\hline$p_{A}$ & incident pressure amplitude & $\rho_{\text {eff }}$ & density of the efective medium \\
\hline$p_{\text {inc }}$ & incident pressure & $\rho_{G}$ & density of the filling gas \\
\hline$p_{\text {inf }}$ & pressure at infinity & $\rho_{L}$ & density of the surrounding fluid \\
\hline$p_{o}$ & atmospheric pressure & $\rho_{S}$ & density of the encapsulating shell \\
\hline$p_{s}$ & scattered pressure & $\sigma_{1}$ & surface tension at the inner microbubble interface \\
\hline$P_{v i s}$ & power dissipated due to viscous friction & $\sigma_{2}$ & surface tension at the outer microbubble shell interface \\
\hline$r$ & radial distance from microbubble center & $\omega$ & circular frequency \\
\hline$R_{1}$ & inner microbubble radius & $\omega_{0}$ & undamped natural frequency \\
\hline
\end{tabular}

where $R_{1}$ and $R_{2}$ are the inner and outer radii, respectively, of the microbubble and $p_{\infty}$ is the pressure at infinity given by $p_{\infty}=p_{o}+p_{A} \operatorname{Sin}(\omega t)$. The remaining symbols are defined in Table I. Further details of the model are given in [3] and [6].

The models for multiple scattering described in the next section are valid for linear scattering behavior only. Initially, therefore, it is necessary to linearize (1) as described in Appendix A. This yields the equation:

$$
\ddot{x}+\delta_{d} \dot{x}+\omega_{o}^{2} x=-\frac{p_{A} \sin (\omega t)}{\rho_{s} R_{o 1}^{2} \alpha},
$$

where

$$
\begin{aligned}
\omega_{o}^{2}= & {\left[3 \kappa p_{o}-\frac{2 \sigma_{1}}{R_{o 1}}-\frac{2 \sigma_{2} R_{o 1}^{3}}{R_{o 2}^{4}}\right.} \\
& \left.+\frac{4 V_{s} G_{s}}{R_{o 2}^{3}}\left(1+z\left(1+\frac{3 R_{o 1}^{3}}{R_{o 2}^{3}}\right)\right)\right]\left(R_{o 1}^{2} \rho_{s} \alpha\right)^{-1}, \\
\alpha= & {\left[1+\left(\frac{\rho_{L}-\rho_{s}}{\rho_{s}}\right) \frac{R_{o 1}}{R_{o 2}}\right], } \\
\delta_{d}= & 4\left(\frac{\mu_{s} V_{s}+\mu_{L} R_{o 1}^{3}}{R_{o 1}^{3} R_{o 2}^{3} \rho_{s} \alpha}\right)+\delta_{\mathrm{rad}}, \\
z= & {\left[\frac{2 \sigma_{1}}{R_{o 1}}+\frac{2 \sigma_{2}}{R_{o 2}}\right] \frac{R_{o 2}^{3}}{4 V_{s} G_{s}} . }
\end{aligned}
$$

The pressure radiated by a microbubble oscillating with low amplitude under the influence of an incident pressure $p_{\text {inc }}=p_{A} \operatorname{Sin}(\omega t)$ may be approximated [7] by:

$$
p_{s}=\frac{\rho_{L} R_{o 1}^{2} \dot{R}_{1} \omega}{r} e^{i\left(\frac{\pi}{2}-k_{L} r\right)} .
$$

Substituting for $\dot{R}$ from the above, this becomes:

$p_{s}=\left(\frac{\rho_{L} R_{o 1}}{\rho_{s} \alpha\left(\left(\frac{\omega_{o}^{2}}{\omega^{2}}-1\right)^{2}+\frac{\delta_{d}^{2}}{\omega^{2}}\right)}\right) \frac{p_{A} i \sin (\omega t+\phi)}{r} e^{-i k_{L} r}$

The validity of the assumptions inherent in this treatment is discussed later.

\section{B. Linear Multiple Scattering in Microbubble Suspensions}

One of the simplest multiple scattering models is that derived by Foldy [8]. The suspension of scatterers is treated as an effective medium. A set of linear equations relating the field scattered by each scatterer to the incident field is derived and expressed in matrix form. Inversion of the matrix of coefficients results in a multiple scattering series, with each term corresponding to a sequence of scatterings. The average Green's function is obtained by taking 
the ensemble average over all possible scatterer configurations, and the corresponding wave number for the effective medium, $K_{\text {eff }}$, may be expressed as:

$$
K_{\text {eff }}^{2}=k_{L}^{2}+4 \pi n f
$$

where $k_{L}=\omega / c_{L}$ is the wave number of the suspending fluid with no scatterers present, $n$ is the scatterer concentration, and $f$ is the scattering function for an individual scatterer. By definition of the scattering function, the scattered pressure from a given scatterer may be expressed as:

$$
p_{s}=f \frac{p_{i n c}(t)}{r} e^{-i k_{L} r}
$$

Comparing with (4), the scattering function for a microbubble is thus:

$$
f=\left(\frac{\rho_{l} R_{01}\left(\left(\frac{\omega_{o}^{2}}{\omega^{2}}-1\right)-i \frac{\delta_{d}}{\omega}\right)}{\rho_{s} \alpha\left(\left(\frac{\omega_{o}^{2}}{\omega^{2}}-1\right)^{2}+\frac{\delta_{d}^{2}}{\omega^{2}}\right)}\right) .
$$

In deriving (5), it was assumed that the mean field incident upon any one scatterer is large compared with the field scattered by its close neighbors and that each scatterer rescatters the field just once. Therefore, the validity of Foldy's model is restricted to relatively low concentration suspensions. A more recent model for multiple scattering that takes account of second order scattering events from each scatterer and, therefore, is valid for higher scatterer concentrations was derived by Henyey [9] and may be expressed as:

$$
\begin{aligned}
K_{\text {eff }}^{2} & =k_{L}^{2}+4 \pi n F \text { where } \\
F & =\left(\frac{1}{f} k_{L}-K_{e f f}\right)^{-1} .
\end{aligned}
$$

The validity of this expression is restricted to the regime $n|f|^{3} \ll 1$ because contributions of this order and beyond are not considered.

Eq. (5) and (8) were used to calculate the attenuation coefficients for microbubble suspensions of varying concentration $^{1}$ and mean radii over a range of insonation frequencies. Properties for the contrast agent Albunex ${ }^{\circledR}$ (Molecular Biosystems Inc., San Diego, CA) as derived by Church [3] were used, as shown in Table II, to enable comparison with previous studies. Assuming linear propagation, the wave number for a given medium is, by definition, complex: $K_{\text {eff }}=\operatorname{Re}\left(K_{\text {eff }}\right)+\operatorname{iIm}\left(K_{e f f}\right)$, and the attenuation coefficient may be found from the imaginary component as:

$$
a=20 \log _{10}(\mathrm{e}) \operatorname{Im}\left(K_{\text {eff }}\right),
$$

\footnotetext{
${ }^{1}$ The concentrations used in the simulations were sufficiently low that dynamic coupling between individual microbubbles would not be expected according to the limits defined by Feuillade [10].
}

where:

$$
\operatorname{Im}\left(K_{e f f}\right)=\sqrt{\left|K_{e f f}^{2}\right|} \sin \left(\frac{\arg \left(K_{e f f}^{2}\right)}{2}\right) .
$$

The same principle may be applied to (8), although, in this case, $\operatorname{Im}\left(K_{e f f}\right)$ must be obtained numerically from the resulting cubic equation.

For comparison, the attenuation coefficient also was found assuming no multiple scattering using the summation method adopted by Medwin [11] for free bubbles whereby:

$$
a=10 \log _{10} e \int_{0}^{\infty} s_{e x t} n\left(R_{1}\right) d R_{1},
$$

here $s_{\text {ext }}$ is the extinction cross section and $n\left(R_{1}\right)$ describes the microbubble size distribution. For a coated microbubble:

$$
s_{e x t}=\frac{\left\langle P_{v i s}\right\rangle_{t}}{I_{i}}=\frac{4 \pi \omega^{2} \rho_{l} c \delta_{d}}{\left(\left(\omega_{o}^{2}-\omega^{2}\right)^{2}+\delta_{d}^{2} \omega^{2}\right) \alpha \rho_{s}},
$$

where \langle\rangle$_{t}$ indicates temporal averaging.

\section{Nonlinear Multiple Scattering}

A number of models for nonlinear multiple scattering in liquids containing free bubbles have been derived. In 1968, Van Wijngaarden [12] presented a heuristic treatment of the problem. A more mathematically rigorous derivation of his results was provided by Caflisch et al. [13] and was subsequently modified by Commander and Prosperetti [14] to include a more realistic treatment of the filling gas. A number of other models (e.g., [15], [16]) also have been presented, but these do not differ fundamentally from the Van Wijngaarden/Caflisch treatment.

None of the existing models consider the effect of an encapsulating shell upon bubble behavior, and the majority of the results presented relate to linear behavior only. The aim of this section is to present a new model, derived according to the reasoning of Van Wijngaarden and Caflisch, which includes the effects of an encapsulating shell and can be solved numerically in order to examine the effects of multiple scattering upon nonlinear microbubble behavior. The derivation is summarized below.

Assuming spherical symmetry, conservation of mass in spherical polar coordinates gives:

$$
\frac{\partial \rho_{e f f}}{\partial t}+\nabla \cdot\left(\rho_{e f f} \mathbf{u}\right)=0
$$

where subscript ef $f$ denotes the effective medium (i.e., fluid + microbubbles), $\rho$ is density, and $\mathbf{u}$ is radial velocity.

The volume fraction of microbubbles in the mixture is:

$$
\beta=\frac{4}{3} \pi R_{2}^{3} n \approx \frac{4}{3} \pi R_{1}^{3} n,
$$


TABLE II

Parameter Values for Suspensions of Albunex ${ }^{\circledR}$ in Water.

\begin{tabular}{lllll}
\hline & Parameter & Symbol & Value & Unit \\
\hline Gas (air) & Polytropic constant & $\kappa$ & 1.0 & \\
& Ambient pressure & $p_{o}$ & 0.1 & $\mathrm{MPa}$ \\
Shell (serum albumin) & Thickness & $d_{e}$ & 15 & $\mathrm{~nm}$ \\
& Density & $\rho_{s}$ & 1100 & $\mathrm{kgm}^{-3}$ \\
& Viscosity & $\mu_{s}$ & 1.77 & $\mathrm{Pas}$ \\
& Shear modulus & $G_{s}$ & 88.8 & $\mathrm{MPa}$ \\
& Surface tension & $\sigma_{1}$ & 0.04 & $\mathrm{Nm}^{-1}$ \\
& & $\sigma_{2}$ & 0.005 & $\mathrm{Nm}^{-1}$ \\
Liquid (water) & Density & $\rho_{1}$ & 1000 & $\mathrm{kgm}^{-3}$ \\
& Viscosity & $\mu_{1}$ & 0.001 & $\mathrm{Pas}$ \\
\hline
\end{tabular}

where $n$ is the number of microbubbles per unit volume. As will be shown below, $n$ may be defined so that (14) is valid for an arbitrary size distribution.

Because $\rho_{L} \gg \rho_{G}$, where $L$ and $G$ denote the suspending liquid and filling gas, respectively, and:

$$
\beta \ll 1 \text { so } \rho_{\text {eff }} \approx \rho_{G} \beta+\rho_{L}(1-\beta) \approx \rho_{L}(1-\beta) .
$$

The contribution from the microbubble shells has been ignored owing to its small value and the fact that $\rho_{L}$ is similar to $\rho_{s}$ (Table II).

Thus:

$$
\frac{1}{\rho_{L}} \frac{\partial \rho_{L}}{\partial t}+\nabla \cdot \mathbf{u}-\frac{1}{1-\beta} \frac{d \beta}{d t}=0
$$

If there is no fragmentation or coalescence of the microbubbles over the time period considered, the number of microbubbles will remain constant and:

$$
\frac{\partial n}{\partial t}+n \nabla \cdot \mathbf{u}_{C}=0
$$

where $\mathbf{u}_{c}$ is the velocity of the microbubble wall.

Differentiating (14) and substituting from (17) gives:

$$
\begin{aligned}
\frac{d \beta}{d t} & =\frac{4}{3} \pi\left(3 R_{1} n \frac{\partial R_{1}}{\partial t}+R_{1}^{3} \frac{\partial n}{\partial t}\right) \\
& =\frac{4}{3} \pi\left(3 R_{1} n \frac{\partial R_{1}}{\partial t}+R_{1}^{3}\left(n \nabla \cdot \mathbf{u}_{c}\right)\right)
\end{aligned}
$$

and substituting this into (16) gives:

$$
\frac{1}{\rho_{L}} \frac{\partial \rho_{L}}{\partial t}+\nabla \cdot \mathbf{u}-\frac{4 \pi}{3(1-\beta)}\left(3 R_{1} n \frac{\partial R_{1}}{\partial t}+R_{1}^{3}\left(n \nabla \cdot \mathbf{u}_{c}\right)\right)=0 .
$$

Because

$$
\begin{aligned}
& O(\mathbf{u}) \approx O\left(\mathbf{u}_{c}\right) \text { so } \beta \nabla \cdot \mathbf{u}_{c} \ll \nabla \cdot \mathbf{u} \text { and thus } \\
& \frac{d \beta}{d t} \approx \frac{\partial \beta}{\partial t} .
\end{aligned}
$$

Eq. (19) may be simplified to:

$$
\frac{1}{\rho_{L}} \frac{\partial \rho_{L}}{\partial t}+\nabla \cdot \mathbf{u}-\frac{\partial \beta}{\partial t}=0
$$

and using $d p / d \rho_{L}=c_{L}^{2}$, this may be expressed as:

$$
\frac{1}{\rho_{L} c_{L}^{2}} \frac{\partial p}{\partial t}+\nabla \cdot \mathbf{u}-\frac{\partial \beta}{\partial t}=0 .
$$

From conservation of momentum:

$$
\begin{aligned}
& \frac{\partial}{\partial t}\left(\rho_{e f f} \mathbf{u}\right)+\nabla \cdot\left(\rho_{e f f} \mathbf{u}\right) \mathbf{u}=\rho_{e f f} \frac{\partial \mathbf{u}}{\partial t} \\
& \quad+\nabla \cdot\left(\rho_{e f f} \mathbf{u}\right) \mathbf{u}-\nabla \cdot\left(\rho_{e f f} \mathbf{u}\right) \mathbf{u} \approx \rho_{L} \frac{\partial \mathbf{u}}{\partial t}=-\nabla p .
\end{aligned}
$$

The effective medium is assumed to be homogeneous and $\rho_{L}$ and $c_{L}$ are assumed to be constant over the range of pressures and frequencies. Thus, using (22) to eliminate $\mathbf{u}$ in (21) gives:

$$
\frac{1}{\rho_{L} c_{L}^{2}} \frac{\partial^{2} p}{\partial t^{2}}-\nabla^{2} p=\frac{\partial^{2} \beta}{\partial t^{2}}
$$

If $\beta$ is defined for an arbitrary size distribution $h\left(R_{o 1}, y\right)$ then:

$$
\beta=\frac{4}{3} \pi \int_{0}^{\infty} R_{1}^{3}\left(R_{o 1}, y, t\right) h\left(R_{o 1}, y\right) d R_{o 1},
$$

and:

$$
\frac{\partial^{2} \beta}{\partial t^{2}}=4 \pi \int_{0}^{\infty}\left(2 R_{1}\left(\frac{\partial R_{1}}{\partial t}\right)^{2}+R_{1}^{2} \frac{\partial^{2} R_{1}}{\partial t^{2}}\right) h\left(R_{o 1}, y\right) d R_{o 1} .
$$

To solve (23), a further equation of motion defining $R_{1}$ is needed. For the case of a coated microbubble, (1) is suitable for this purpose, although it is appropriate at this point to discuss its validity a little further.

In (1) the surrounding fluid has been modeled as a simple Newtonian liquid with viscosity $\mu_{L}$. It has been shown in previous work $([6],[16])$ that the surrounding fluid has a small influence upon microbubble behavior compared with that of the encapsulating shell ${ }^{2}$. Moreover, owing to their low compressibility, blood cells are poor backscatterers of ultrasound relative to microbubbles. Thus, given

\footnotetext{
${ }^{2}$ This does not apply to the case of a microbubble enclosed in a narrow blood vessel, which is not considered.
} 
that the main concern of this study was multiple scattering from microbubbles, the advantages of using a more advanced model for the surrounding fluid were considered to be small.

On similar grounds, the encapsulating shell has been modeled as a uniform linear viscoelastic coating. This treatment is valid for the small amplitude oscillations of polymeric shells considered in the majority of this study. It is questionable whether this is strictly the case at the larger amplitudes considered in the work on higher insonation pressures [6]. However, in the absence of reliable material data for these conditions, it was judged that the linear model would provide a reasonable approximation; and again, the main interest of the work was multiple scattering of the radiated field. The problem of shell modeling at high-insonation pressures will be addressed in future work.

It has been assumed that the filling gas would behave isothermally during microbubble compression and expansion, i.e., $\kappa=1$. This is reasonable because it was assumed in deriving (1) that the surface tension forces would be balanced by the strain in the encapsulating shell [c.f. the definition of $z$ in (2)]. Thus, the thermal diffusivity of the gas, and hence $\kappa$, should remain constant during oscillation. The thermal gradient inside the microbubble will be negligible at the frequencies of oscillation considered (1-10 MHz) owing to the high specific heat capacity of plasma $\left(3800 \mathrm{~kJ} / \mathrm{kg}^{\circ} \mathrm{C}\right)$ and the small size of the microbubble $(2-8 \mu \mathrm{m}$ diameter). Consistent with this assumption, thermal damping also has been neglected. The validity of this approach may be confirmed by examining the relative size of the thermal and viscous damping factors for a microbubble with an albumin shell [3] under the relevant insonation conditions. It should be noted, however, that thermal damping may not always be negligible for microbubbles, in particular if the encapsulating shell has a relatively small influence upon microbubble oscillations. Further discussion may be found in [17].

Acoustic radiation damping has been accounted for in (1). The term $b_{\text {rad }}$ represents an acoustic damping factor that may take the form of the compressibility terms derived by Keller and Miksis [18] or a simpler formulation such as that used by Medwin [11] and de Jong et al. [2]. For the purposes of this preliminary study, however, acoustic damping has been neglected $\left(b_{\mathrm{rad}}=0\right)$. This is consistent with the assumption of constant density in the surrounding fluid and is a reasonable approximation for microbubble behavior at the relatively low concentrations and insonation pressures considered. For these conditions, the ratio of microbubble wall velocity to sound speed in the effective medium always will be much less than unity, and the value of $b_{\text {rad }}$, therefore, will be negligible compared with the viscous dissipation term. Again, this is discussed in more detail in [17]. Clearly, however, if the model is to be developed to simulate higher pressures and concentrations, this will no longer be the case, and methods for including acoustic damping in the model will be discussed shortly.
It should be noted that this approach is similar in some respects to that adopted in a recent treatment of free bubbles [19]. However, the work presented here was completed independently prior to publication of the above; and although both treatments follow the reasoning of Van Wijngaarden [12], Caflisch [13], and Commander and Prosperetti [14] in their description of the effective medium, they differ fundamentally in their description of the scatterers. The present work considers microbubbles surrounded by encapsulating shells, which, as mentioned above, behave very differently from free bubbles having diameters of a few millimeters.

\section{Numerical Solution}

The solution for the simple case of a monodisperse, homogeneous microbubble suspension was considered in one dimension. Eq. (1) is coupled to $(23)$ via $p_{\infty}(t)$, the pressure in the effective medium. Therefore, (23) must be discretized so that the pressure $p_{i}$ is evaluated at each point $y_{i}$ along the direction of transmission:

$$
\frac{1}{c_{L}^{2}} \frac{\partial^{2} p_{i}}{\partial t^{2}}-\nabla^{2} p_{i}=4 \rho_{L} n \pi\left[2 R_{1} \dot{R}_{1}^{2}+R_{1}^{2} \ddot{R}_{1}\right]_{i}
$$

Replacing the derivatives by standard finite difference approximations [20] gives:

$$
\begin{array}{r}
\frac{p_{j+1, i}-2 p_{j, i}+p_{j-1, i}}{c_{L}^{2} \Delta t^{2}}-\frac{p_{j, i+1}-2 p_{j, i}+p_{j, i-1}}{\Delta y^{2}}= \\
4 \rho_{L} n \pi\left[2 R_{1} \dot{R}_{1}^{2}+R_{1}^{2} \ddot{R}_{1}\right]_{j, i} .
\end{array}
$$

Subscripts $i$ and $j$ represent discretization in space and time, respectively.

Rearranging (27) for $p_{j, i}$ gives:

$$
\begin{aligned}
p_{j, i}= & 2 p_{j-1, i}-p_{j-2, i} \\
& +c_{L}^{2} \Delta t^{2}\left(\frac{p_{j-1, i+1}-2 p_{j-1, i}+p_{j-1, i-1}}{\Delta y^{2}}\right) \\
& \quad+4 \rho_{L} n \pi c_{L}^{2} \Delta t^{2}\left[2 R_{1} \dot{R}_{1}^{2}+R_{1}^{2} \ddot{R}_{1}\right]_{j-1, i}
\end{aligned}
$$

Eq. (1) and (28) were nondimensionalized according to the following scheme:

$$
\begin{aligned}
& {[\text { mass }]=\left[p_{o} R_{o 1} / \omega^{2}\right][\text { length }]=\left[R_{o 1}\right]} \\
& {[\text { time }]=[1 / \omega]}
\end{aligned}
$$

Eq. (28) was evaluated using purpose written code simultaneously implementing a fourth order Runge-Kutta procedure for solving (1) at each point in space and time.

Initial conditions representing an initially quiescent fluid were taken as:

$$
p_{0, i}=p o \text { and } p_{1, i}=p_{o}
$$


Boundary conditions representing a plane source of sound at $y=0$ and undisturbed fluid at $y=N \Delta y$. were taken as:

$$
p_{j, 0}=p_{o}+p_{A} \text { and } p_{j, N}=p_{o} .
$$

$N$ was selected to ensure that $N \Delta y$ was greater than the distance propagated by the wave during the time period $M \Delta t$ considered. Similarly the latter was selected so that $M \Delta t$ was greater than the pulse length.

The model of the imaging pulse, containing $m=$ five cycles, was based on a generalized version of the model given by $[21]$ :

$$
\begin{gathered}
\text { for } 0 \leq \frac{\omega t}{m} \leq 2 \pi, p_{\text {inc }}(t)= \\
p_{A}\left(1-\cos \left(\frac{\omega t}{m}\right)\right) p_{a}\left(1-\sin \left(\frac{\omega t}{m}\right)\right) \cos (\omega t) \sin (\omega t) .
\end{gathered}
$$

The results were processed in Matlab ${ }^{\circledR}$ (The MathWorks, Natick, MA) in order to obtain the attenuation coefficients corresponding to a range of different frequencies, insonation pressures, microbubble radii, and concentrations.

It was necessary to consider both accuracy and computation time in selecting $\Delta y$. For the sake of the former, it was necessary for $\Delta y$ to be smaller than the smallest wavelength considered $\left(\approx 1.5 \times 10^{-4} \mathrm{~m}\right)$ and for the subsequent selection of $\Delta t$ to satisfy the Von Neumann criterion [20]. Therefore, the maximum values for $\Delta y$ and $\Delta t$ were chosen to be $1.5 \times 10^{-5} \mathrm{~m}$ and $1.0 \times 10^{-8} \mathrm{~s}$, respectively, and these were reduced proportionately until the difference between the results for $\Delta y$ and $\Delta t$ and $\Delta y / 2$ and $\Delta t / 2$ was found to be less than $2 \%$. It was recognized that this method only provided a first order means of ensuring numerical stability. Nevertheless, its validity was supported by making further checks of the results for $\Delta y / 4$ and $\Delta t / 4$, and for different transmission distances (i.e., different values of $M$ and $N$ ) to ensure that the differences in the calculated attenuation coefficients remained small. The agreement with the results from linear theory, described below, also provided corroboration.

To enable single and multiple scattering models to be compared at different pressures, results were plotted from (28) and from a numerical solution of (1). For the latter, attenuation was found from (11) with $s_{\text {ext }}$ being found from numerical integration of the dissipated power over the course of a single pulse:

$$
\begin{gathered}
s_{\text {ext }}=\frac{\left\langle P_{v i s}\right\rangle_{t}}{I_{i}} \text { where } P_{v i s}=P_{s, v i s}+P_{L, v i s}, \\
P_{s, v i s}=\int_{R_{1}}^{R_{2}} \frac{-48 \pi \mu_{s} R_{1}^{4} \dot{R}_{1}}{r^{4}} d r=\frac{16 \pi \mu_{s} V_{s} R_{1} \dot{R}_{1}^{2}}{R_{2}^{3}}, \\
P_{L, v i s}=\int_{R_{2}}^{\infty} \frac{-48 \pi \mu_{L} R_{1}^{4} \dot{R}_{1}}{r^{4}}=\frac{16 \pi \mu_{L} R_{1}^{4} \dot{R}_{1}^{2}}{R_{2}^{3}} .
\end{gathered}
$$

Further details of this treatment may be found in [21].

\section{EXPERIMENTAL INVESTIGATION}

As mentioned in the introduction, experimental evidence for multiple scattering in ultrasound contrast agent suspensions already has been reported. Marsh et al. [4] performed attenuation and phase velocity measurements in suspensions of Albunex ${ }^{\circledR}$ and found that the peak attenuation was underestimated by (11) for suspensions with concentrations greater than $10^{6}$ particles per milliliter. There are, however, a number of factors to consider in assessing the reliability of this evidence. For example, the measurements were reported as being extremely sensitive to temperature, and the heat generated during the experiment would have been expected to increase with increasing concentration. Thus, there would have been a variation in the attenuation of the different test samples regardless of whether or not multiple scattering was taking place. From existing data [23], the additional heating would, in fact, have been expected to reduce the measured attenuation, but a separate experiment to confirm that this would be the case in Albunex ${ }^{\circledR}$ suspensions would be needed to remove the uncertainty.

In comparing their experimental results with theory, Marsh et al. [4] assumed that the Albunex ${ }^{\circledR}$ microbubbles would behave linearly. However, at the insonation pressures used (0.2-0.3 MPa), perceptible nonlinear behavior would have been expected, and this would have increased the apparent attenuation at the fundamental frequency. It is not clear that this could explain the enhancement in attenuation at higher concentrations, however, because, in the absence of multiple scattering, the generation of higher harmonics should not be a function of concentration. The values of the shell parameters derived by Marsh et al. [4] were also rather different from those derived for the same agent (Albunex ${ }^{\circledR}$ ) by de Jong et al. [2] (8000 versus $4200 \mathrm{dyn} / \mathrm{cm}$ for shell stiffness and 0.004 versus 0.0054 Pas for shell viscosity). They were, moreover, found to be inconsistent between different sets of results from the same experiment.

These discrepancies inevitably call into question the reliability of the model and/or the experimental technique, and hence that of the results and their interpretation. Thus, additional results are required for corroboration. Owing to the uncertainty in the value of microbubble shell parameters and in their uniformity within a given population, it would be impractical to attempt a quantitative comparison between the theoretical and experimental results. However, it should be possible to determine the nature of the relationship between concentration and attenuation in microbubble suspensions without these values. This was the aim of the experiment described below.

\section{A. Apparatus}

In terms of objective, the experiment was similar to that conducted by Marsh et al. [4]. Broadband transmission measurements were made of the attenuation of ultrasound in microbubble suspensions of varying concentration. There were a number of differences in the apparatus 


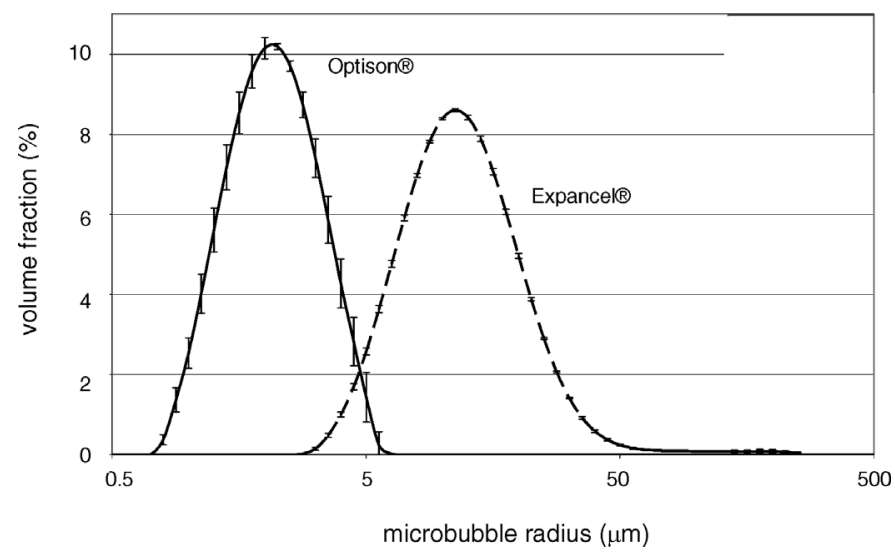

Fig. 1. Size distribution data for Optison ${ }^{\circledR}$ and Expancel ${ }^{\circledR}$.

and procedure, however, which were introduced with the aim of reducing uncertainty.

Two different types of coated microbubbles were used: a commercial diagnostic contrast agent, Optison ${ }^{\circledR}$ (Amersham PLC, Bucks, UK) and a blowing agent ${ }^{3}$ Expancel $^{\circledR}$ (Casco Products AB, Sundsvall, Sweden). Their respective properties are shown in Table III. Optison ${ }^{\circledR}$ was selected as having similar shell properties, but greater stability, than Albunex ${ }^{\circledR}$ owing to its containing a gas of higher molecular weight than air. It was intended that this should lessen the handling problems experienced by Marsh et al [4]. The aim in selecting Expancel ${ }^{\circledR}$ was to examine whether similar behavior would be observed with microbubbles having a different coating material and size distribution. Size distribution measurements were performed using a Malvern Mastersizer (2000 series, Malvern, UK). The results for each type of microbubble are shown in Fig. 1.

The measurements were made using a pair of broadband unfocused transducers (5108R, Olympus NDT, Waltham, MA) each having a nominal center frequency of $5 \mathrm{MHz}$, diameter $19 \mathrm{~mm}$, and a $3 \mathrm{~dB}$ bandwidth of $3 \mathrm{MHz}$. As shown in Fig. 2, the transducers were activated using a pulser/receiver unit (5055 PR, Olympus NDT) that was set to give a transmitted peak negative pressure in water of approximately $50 \mathrm{kPa}$. The output pressure from the transducer was measured using a needle hydrophone (698, Precision Acoustics Ltd., Dorchester, Dorset, UK) with a $0.2 \mathrm{~mm}$ tip that was scanned across the transducer face at different axial distances to determine the beam profile. The angular tolerance for the hydrophone was $0 \mathrm{~dB}$ for $\pm 10^{\circ}$ over the measured bandwidth. The signals from the transducer were captured using a digital oscilloscope (LeCroy 9310M Dual 300 MHz, LeCroy Ltd., Abingdon, Oxon., UK) connected to a computer.

The pressure used was lower than that selected by Marsh et al. [4], and, according to the results of previous simulations [6], should have been low enough both to avoid rapid microbubble destruction and reduce the pres-

\footnotetext{
${ }^{3}$ Polymer encapsulated gas bubbles, similar to microbubbles, but used to increase the bulk of polymer melts for various manufacturing processes.
}

ence of significant higher harmonics in the microbubble response. This was desirable in order to minimize the time and pressure dependence of the attenuation measurements and because low insonation pressures corresponded to the behavior considered in the first part of the modeling work. It also reduced the likelihood of distortion of the results due to nonlinear propagation, which would have increased the harmonic content of the received signal. The absence of significant harmonic content was verified by examination of the signal frequency spectra. Likewise, the lack of variation in the received signal over the measurement time confirmed that microbubble destruction and drift also was minimal. If the experiments were repeated after the suspensions had been left standing for several minutes, a reduction in the measured attenuation was seen. Consequently, care was taken to ensure that the time between preparing the suspensions and capturing the signals was kept constant and as short as possible.

In order to reduce the effect of temperature fluctuations, all the measurements were conducted at room temperature in a large water bath (length $45 \mathrm{~mm}$, width $25 \mathrm{~mm}$, height $25 \mathrm{~mm}$ ). The instruments used for handling the test samples also were kept immersed in the bath when not in use. A container was designed to hold the microbubble suspensions (Fig. 2). This was constructed from a section of polymethylmethacrylate tubing (inner diameter $60 \mathrm{~mm}$, wall thickness $5 \mathrm{~mm}$, length $30 \mathrm{~mm}$ ) with acoustic "windows" made with polyethylene film at either end. The tubing was held between two square aluminium plates into which recessed circular holes had been cut to allow access to the ends of the tubing and hold it in place. The plates were bolted together to ensure a tight seal. A hole was cut in the wall of the tubing and sealed with a Newplast ${ }^{\circledR}$ (Newclay, Newton Abbot, UK) plug in which two polyethylene tubes were embedded to enable the container to be filled and emptied. The container was located on the base of the tank between two fixed blocks to ensure accurate positioning for each test.

Holders for the transducers also were constructed. The holder for the receiving transducer was positioned on the base of the tank at a known distance from the suspension container. The transmitting transducer was connected to a precision scanning rig to enable accurate positioning with respect to the container and receiver. The distance between the transducers and the container was measured in two ways to reduce the risk of error. First, the number of revolutions of the leadscrew from a known starting position on the scanning rig was recorded. Second, a direct measurement was made using Vernier callipers. The holder for the transmitter was designed to enable the axes of the transducers to be aligned. The correct alignment was determined prior to the experiment by adjusting the position of the transmitter in water until the amplitude of the received signal was maximized.

\section{B. Procedure}

The apparatus was set up as shown in Fig. 2. Each complete set of experiments was performed on the same 
TABLE III

Manufacturer Specifications For Optison ${ }^{\circledR}$ AND EXPAnCel ${ }^{\circledR}$.

\begin{tabular}{lll}
\hline & Optison $^{\circledR}$ & Expancel ${ }^{\circledR}$ \\
\hline Shell material & Human serum albumin & Copolymer (acrylonitrile; vinylidene chloride) \\
Filling gas & Octafluoropropane & Isobutane \\
Mean radius & $2.25 \mu \mathrm{m}$ & $12 \mu \mathrm{m}$ \\
Maximum radius & $16 \mu \mathrm{m}(93 \%$ less than $10 \mu \mathrm{m})$ & $100 \mu \mathrm{m}$ \\
\hline
\end{tabular}

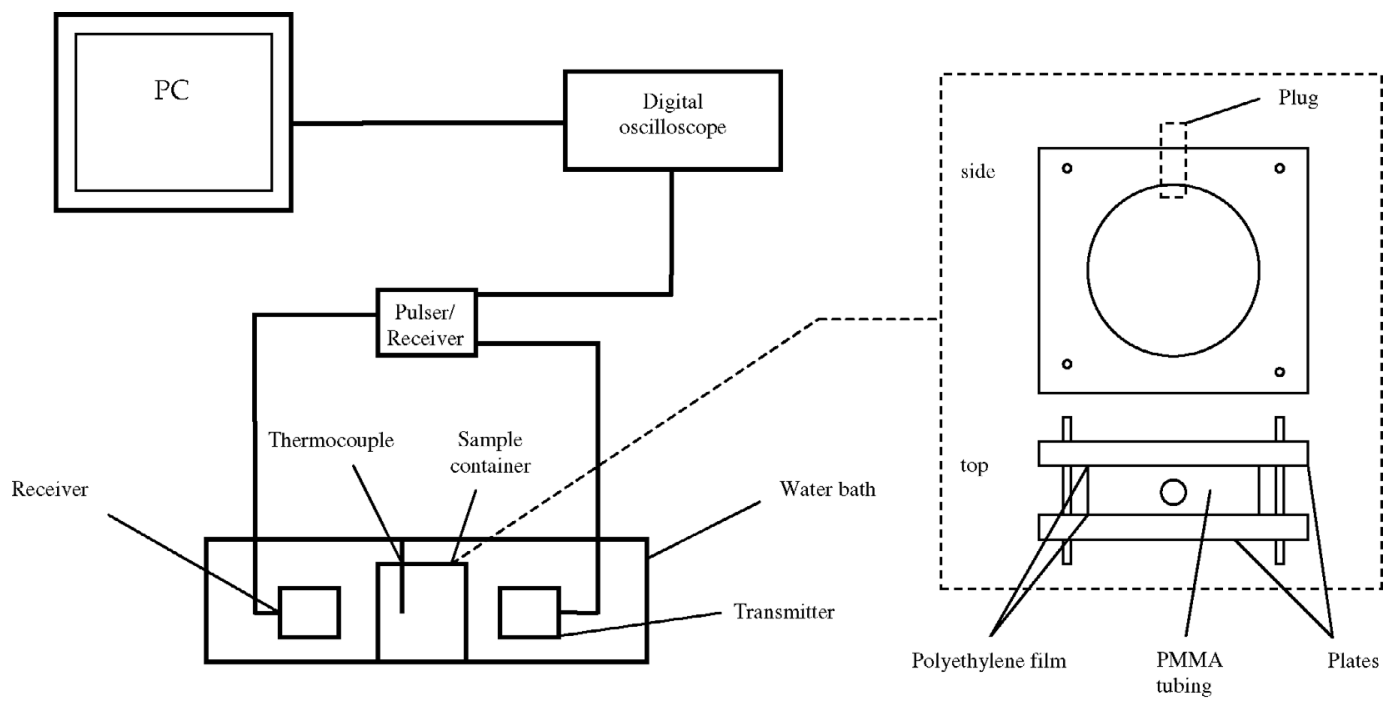

Fig. 2. Schematic of the experimental apparatus, with detail of sample chamber.

day in order to minimize the influence of external factors. The Optison ${ }^{\circledR}$ suspensions were prepared according to the manufacturer's instructions on the product insert. The Expancel ${ }^{\circledR}$ suspensions were prepared by dispersing a known volume $\left(1.55 \times 10^{-4} \mathrm{~m}^{3}\right)$ of product in $300 \mathrm{ml}$ distilled water. The measurement container also was filled with distilled water at room temperature and a 1-ml syringe with a 20 -gauge needle was used to transfer the appropriate volumes of each suspension to the container to yield the concentrations indicated in the results. A fine wire with a looped end was inserted into the container via one of the polyethylene tubes to enable the suspension to be gently stirred. This was done continuously while the measurements were being taken. The temperature in the container also was monitored continuously using a digital thermometer (RS 650-419, RS Components Ltd., Northants, UK).

Broadband pulses were transmitted at a pulse repetition frequency of $0.6 \mathrm{kHz}$ and captured at $10^{8}$ samples per second. For each test, measurements were made first in the water bath without the container present, then through the suspension. This was done in order to reduce further the influence of effects such as temperature fluctuations upon the results. Three pulses were captured for each concentration, and the process was repeated with fresh samples to give a total of six measurements for each test. The chamber was rinsed thoroughly with distilled water in between tests. The time for the pulse to be reflected back to the transmitting transducer also was measured in order to determine velocity.

The captured signals were processed in Matlab ${ }^{\circledR}$. The mean value for each signal was found and subtracted from it in order to remove the dc component. Its frequency spectrum was then obtained via fast Fourier transform. The amplitudes at 4.0, 5.0, and $6.0 \mathrm{MHz}$ were determined, and the attenuation coefficient was found as:

$$
a=\frac{20 \log _{10} e}{d} \ln \left|\frac{A_{1}}{A_{2}}\right|,
$$

where $A_{1}$ is the average amplitude in water for that frequency, $A_{2}$ is the average amplitude with the container present, and $d$ is the length of the container. The mean average attenuation coefficient and corresponding standard deviation then were found for each concentration, from which the experimental uncertainty was estimated.

Similarly, the acoustic velocity for each test with and without the container present was found as:

$$
c_{L}=\frac{d+y_{1}+y_{2}}{t_{2}-t_{1}}, \quad \text { in water }
$$

and:

$$
c_{e f f}=\frac{d}{t_{2}-t_{1}-c_{L}\left(y_{1}+y_{2}\right)}, \text { in the suspension }
$$




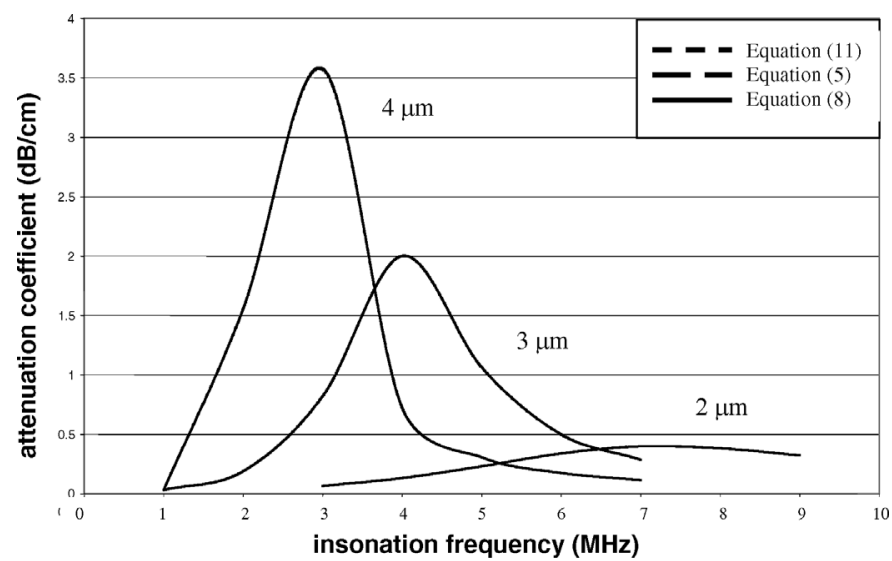

Fig. 3. Variation in attenuation with frequency and microbubble radius for Albunex ${ }^{\circledR}$ suspensions in water with concentrations of $10^{4}$ microbubbles per milliliter, as predicted by (5) (first order multiple scattering), (8) (second order multiple scattering), and (11) (single scattering).

where $t_{2}-t_{1}$ is the separation in time between the first and second received pulses, and $y_{2}$ and $y_{1}$ are the distances between the container and the two transducers. Again, the mean average and standard deviation were calculated for each set of measurements.

It was intended, initially, that a diffraction correction factor should be applied to the results. However, the differences in the measured velocities for water and for the suspensions (Table IV) were found to be too small compared with the experimental uncertainty to justify this. This was somewhat unexpected, given the variation in the values of $\left.\operatorname{Im}\left(K_{e f f}\right)\right)$ from (5) and (8) at the higher concentrations. However, because these equations were derived on the assumption that the velocity would remain constant and equal to $c_{L}$, this was not an accurate means of predicting the variation in velocity. Moreover, given that the transmission distances were small compared with the near field distance, the magnitude of the correction factors would have been relatively small.

\section{Results AND Discussion}

\section{A. Linear Modeling}

Fig. 3 indicates that, at a concentration of $10^{4} \mathrm{mi}-$ crobubbles per milliliter, the effects of multiple scattering should be negligible, and (5), (8), and (11) are then of equal value provided small amplitude linear behavior can be assumed. This would appear to be supported by the existing experimental evidence [4] as will be discussed below. At $10^{5}$ microbubbles per milliliter (Fig. 4) there is some discrepancy between the predictions of (11) and those of (5) and (8) at the peak of the curve for the largest microbubble radius $\left(R_{o 1}=4 \mu \mathrm{m}\right)$. This implies that a small degree of multiple scattering may be expected between the larger microbubbles in the distribution at this concentration, when they are excited at their resonance frequencies.

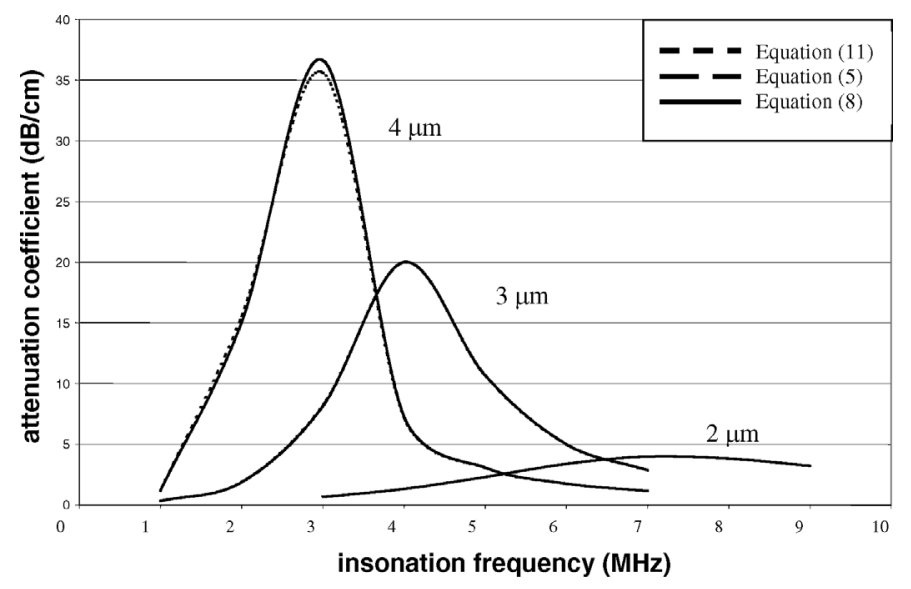

Fig. 4. Variation in attenuation with frequency and microbubble radius for Albunex ${ }^{\circledR}$ suspensions in water with concentrations of $10^{5}$ microbubbles per milliliter, as predicted by (5) (first order multiple scattering), (8) (second order multiple scattering), and (11) (single scattering).

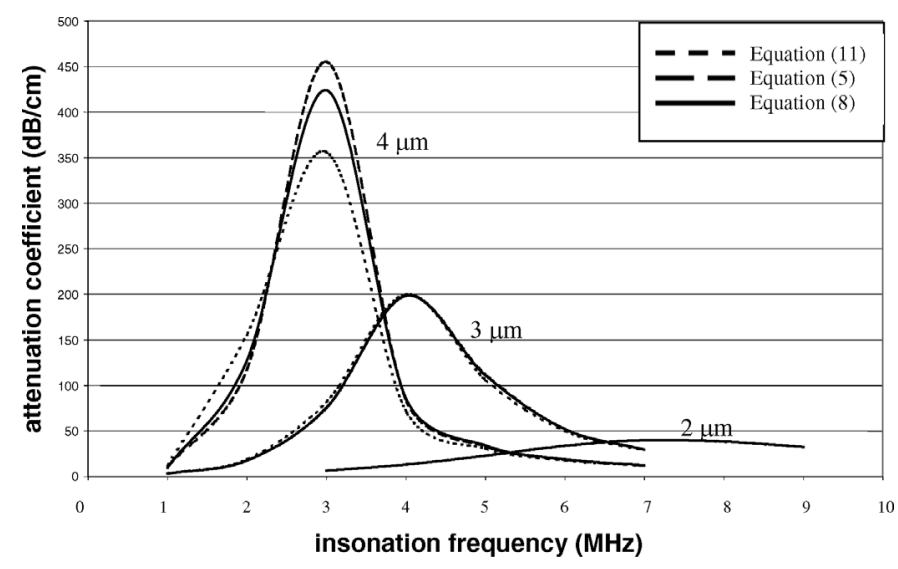

Fig. 5. Variation in attenuation with frequency and microbubble radius for Albunex ${ }^{\circledR}$ suspensions in water with concentrations of $10^{6}$ microbubbles per milliliter, as predicted by (5) (first order multiple scattering), (8) (second order multiple scattering), and (11) (single scattering).

At $10^{6}$ microbubbles per milliliter (Fig. 5) the discrepancies between the curves for (11), (5), and (8) are much more significant, implying that there would be a higher incidence of multiple scattering over a wider range of microbubble radii. Moreover, the differences between the results for (5) and (8), which were negligible at $10^{5}$ microbubbles per milliliter, indicate that higher orders of scattering also may be expected. In this case (5) overestimates the attenuation compared with (8). Physically, this may be explained in terms of the effect of multiple scattering upon the incident field "seen" by individual scatterers and their subsequent response. The initial concentrations of contrast agent suspensions, as supplied by their manufacturers, are of the order of $10^{9}$ microbubbles per milliliter. Clearly the suspensions will be diluted upon injection into the body, and this will be discussed further later, but it would appear that the possibility of multiple scattering cannot be dismissed without further inves- 
TABLE IV

Acoustic Velocity Measurements for Optison ${ }^{\circledR}$ And Expancel ${ }^{\circledR}$ Suspensions.

\begin{tabular}{cccccc}
\hline $\begin{array}{c}\text { Expancel } \\
\text { concentration } \\
(\text { microbubbles } / \mathrm{ml})\end{array}$ & $\begin{array}{c}\text { Mean } \\
\text { velocity } \\
(\mathrm{m} / \mathrm{s})\end{array}$ & $\begin{array}{c}\text { Standard } \\
\text { deviation } \\
(\mathrm{m} / \mathrm{s})\end{array}$ & $\begin{array}{c}\text { Optison }{ }^{\circledR} \\
\text { concentration } \\
\text { (microbubbles } / \mathrm{ml})\end{array}$ & $\begin{array}{c}\text { Mean } \\
\text { velocity } \\
(\mathrm{m} / \mathrm{s})\end{array}$ & $\begin{array}{c}\text { Standard } \\
\text { deviation } \\
(\mathrm{m} / \mathrm{s})\end{array}$ \\
\hline 0 & 1505.4 & 1.7 & 0 & 1506.7 & 2.1 \\
121350 & 1506.2 & 2.2 & 5050 & 1504.7 & 3.0 \\
242700 & 1497.3 & 3.0 & 50500 & 1505.5 & 2.2 \\
364000 & 1498.1 & 2.6 & 707200 & 1505.9 & 2.4 \\
485350 & 1497.3 & 2.2 & 1010300 & 1506.3 & 3.1 \\
606700 & 1500.1 & 3.1 & 2020500 & 1507.5 & 1.8 \\
\hline
\end{tabular}

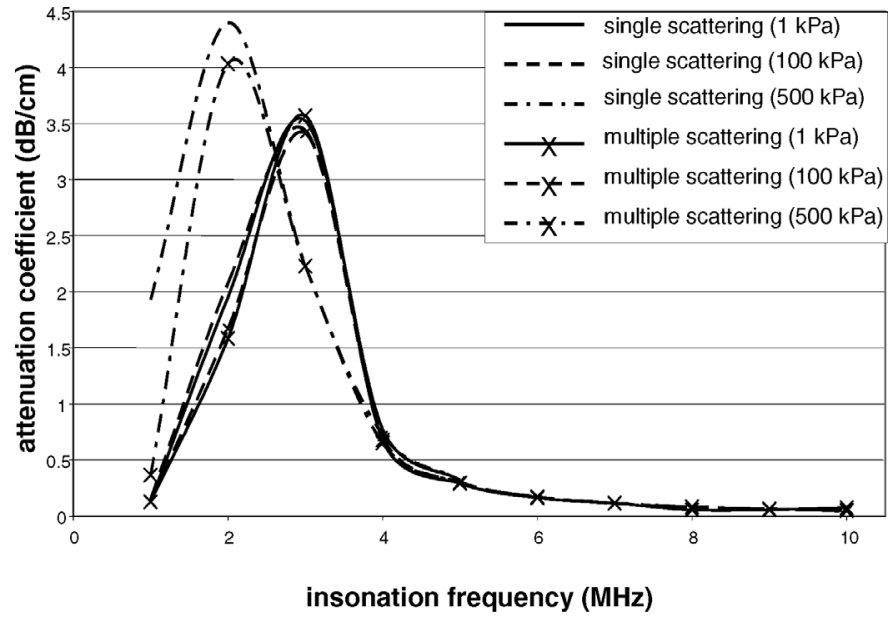

Fig. 6. Comparison between single and multiple scattering models (11) and (28) for Albunex ${ }^{\circledR}$ suspensions of $4 \mu \mathrm{m}$ radii microbubbles in water with concentrations of $10^{4}$ microbubbles per milliliter. Variation in attenuation with insonation frequency and pressure.

tigation. First, however, the nonlinearity of microbubble behavior must be taken into account.

\section{B. Nonlinear Modeling}

As expected, the results for the lower insonation pressures mimicked those shown in Figs. 3, 4, and 5 (solid and dashed lines) for linear scattering. With increasing pressure, however, the results from the single scattering model (11) showed a downward shift in the frequency at which the attenuation was maximized and an increase in the value of the maximum (plain dot-dash line in Figs. 6 and 7). This may be attributed to the increase in prominence of the nonlinear components of the microbubble response with increasing pressure as observed by MacDonald et al. [24]. The same effect also was seen in the results from the multiple scattering model (crossed dot-dash line in Figs. 6 and 7). However, it was considerably less pronounced than was the case for the single scatterer model. This may be explained in terms of the modification of the incident field "seen" by each microbubble as a result of multiple scattering, as mentioned above. Qualitatively, the presence of harmonics in the fields radiated by neighboring microbubbles would skew the incident spectrum toward higher fre-

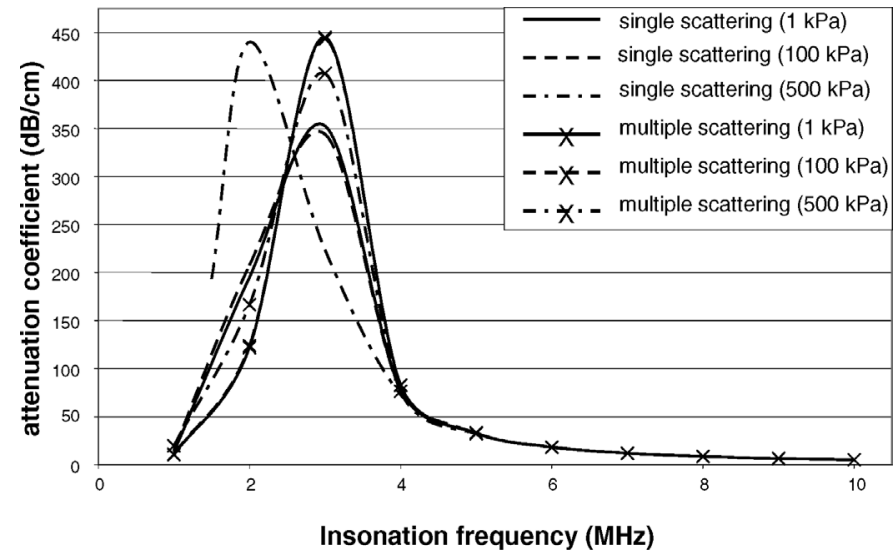

Fig. 7. Comparison between single and multiple scattering models (11) and (28) for Albunex ${ }^{\circledR}$ suspensions of $4 \mu \mathrm{m}$ radii microbubbles in water with concentrations of $10^{6}$ microbubbles per milliliter. Variation in attenuation with insonation frequency and pressure.

quencies. This would counteract the downward shift due to increasing pressure. The potential consequences of using single scatterer models to interpret experimental results are discussed later.

The new model overcomes several of the limitations associated with (5), (8), and (11) for linear scattering. It is valid for the same range of concentrations as (5). Thus, unlike (11), it may be used to predict microbubble behavior when multiple scattering effects are observable. Unlike (5) and (8), however, it is valid for insonation pressures at which microbubbles exhibit nonlinear behavior. In addition, the treatment of the pressure radiated by the microbubble is more realistic than that given by (3), which models the microbubble as a rigid sphere and, therefore, is only valid at very low amplitudes of oscillation [7]. It also enables the effects of different types of insonation (e.g., different pulse shapes) to be examined because it must be solved numerically.

Eq. (28) is still limited in terms of the maximum concentration for which it can be used. For the assumption of an effective medium to be valid, it is necessary to assume that the average pressure field incident upon any one microbubble is large compared with that radiated by its immediate neighbor. Assuming it is valid to take $n^{-1 / 3}$ as 
TABLE V

Limiting Concentrations for Which (28) is VAlid.

\begin{tabular}{cc}
\hline $\begin{array}{c}\text { Radius } \\
\text { (microns) }\end{array}$ & $\begin{array}{c}\text { Limiting concentration } \\
\text { (microbubbles } / \mathrm{ml})\end{array}$ \\
\hline 2 & $6.87 \times 10^{8}$ \\
3 & $3.34 \times 10^{7}$ \\
4 & $3.91 \times 10^{6}$ \\
\hline
\end{tabular}

the average distance between microbubbles, then for the linear case this imposes the following condition [14]:

$$
\frac{\omega R_{o 1}}{n^{-1 / 3} \sqrt{\left(\left(\omega_{o}^{2}-\omega^{2}\right)^{2}+4 \delta_{d}^{2} \omega^{2}\right)}} \ll 1 .
$$

This may be used as an approximate gauge for the validity of (28). Clearly the condition is most severe at the resonance frequency when $\omega=\omega_{o}$ for a given $R_{o}$. The limiting concentrations for each microbubble radius are shown in Table V.

Eq. (28) is analogous to (5) in that only one additional scattering from each microbubble is considered. And (8) is more valid for higher concentrations than either (5) or (28) because it considers higher orders of scattering. For (28) to be valid at higher concentrations, it would be necessary to estimate the speed of sound for the effective medium, rather than treat it as a constant equal to the sound of speed in the surrounding fluid. This could be done iteratively, improving the estimate for $c_{e f f}$ upon each run of the model. For consistency, it also would be necessary to include an acoustic damping factor in (1), which would itself depend upon the sound speed in the effective medium. Before this work is carried out, however, it is important to determine whether the effects of multiple scattering indicated by the above results can be observed experimentally, and hence whether the additional complexity and computing time is in fact warranted.

\section{Experimental Investigation}

As shown in Fig. 8, attenuation was seen to vary linearly with concentration for Optison ${ }^{\circledR}$ over the $3 \mathrm{~dB}$ bandwidth at an insonation pressure of $50 \mathrm{kPa}$. The deviation from a linear fit is shown in Fig. 8. This might have been expected from the theoretical results shown in the previous section. Assuming that the shell parameters for Optison ${ }^{\circledR}$ would be similar to those determined for Albunex ${ }^{\circledR}$ (Table II), the majority of the Optison ${ }^{\circledR}$ microbubbles would have been smaller than the resonant size for the range of frequencies used. Consequently, the effects of multiple scattering would have been small. Indeed, as shown in Fig. 5, the discrepancy between the results from the single and multiple scattering models is practically negligible at resonance for the $2 \mu \mathrm{m}$ radius microbubbles, even at the highest concentrations. These findings are in agreement with the results obtained by Marsh et al. [4] for Albunex ${ }^{\circledR}$ at nonresonant frequencies.

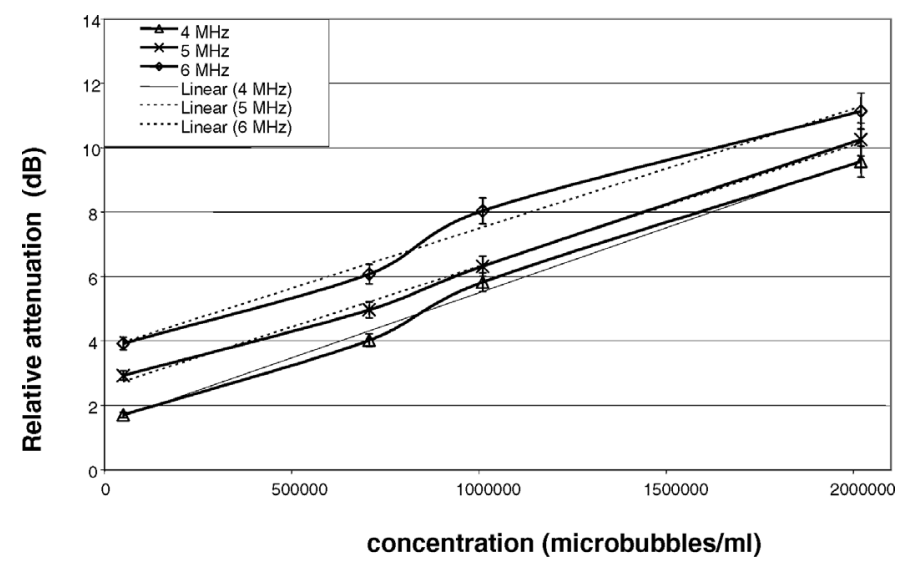

Fig. 8. Variation in attenuation with concentration for suspensions of Optison ${ }^{\circledR}$ in distilled water.

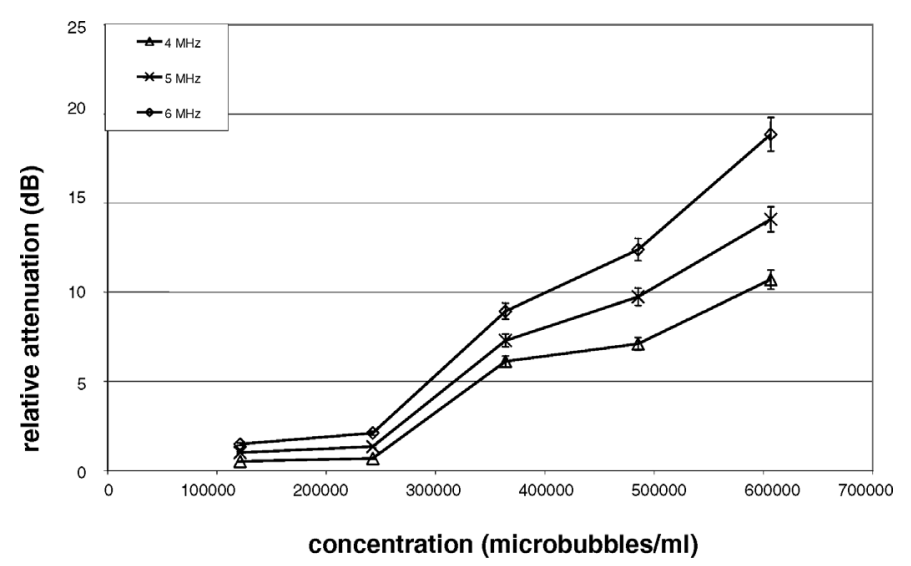

Fig. 9. Variation in attenuation with concentration for suspensions of Expancel ${ }^{\circledR}$ in distilled water.

As will be discussed shortly, the results for Optison ${ }^{\circledR}$ were intended to be representative of diagnostic conditions for this agent. The aim in repeating the experiment with Expancel ${ }^{\circledR}$ was to investigate whether multiple scattering could be observed with microbubbles having different shell properties and larger diameters. As shown in Fig. 9, the relationship between attenuation and concentration for Expancel ${ }^{\circledR}$ was found to be nonlinear. Attenuation was found to increase at a rate that increased with concentration, as predicted by (5), (8), and (28). The shell parameters for Expancel ${ }^{\circledR}$ are not known at present. Nevertheless, the results would appear to indicate that at least a proportion of the population were resonant over the range of frequencies used. This again concurs with the results of Marsh et al. [4] who found that the attenuation in Albunex ${ }^{\circledR}$ suspensions was underpredicted by single scattering models over the resonance regime.

Notwithstanding the qualitative agreement between the experimental and theoretical results, there remains a degree of uncertainty that warrants further discussion. As mentioned above, it was assumed that the shell parameters for Optison ${ }^{\circledR}$ and Albunex ${ }^{\circledR}$ would be similar, given that they describe the same material. However, further investigation is required in order to confirm this and to de- 
termine the appropriate values for Expancel ${ }^{\circledR}$. Similarly, it was necessary to assume that any variability in the shell parameters would be constant between samples. The similarity between the results from different samples indicated that this assumption was valid. It should, however, be verified, because such variability could account for the differences in the response of individual microbubbles observed by Postema et al. [25] and could contribute to the pressure dependence of attenuation in microbubble suspensions. The microbubble concentration $n$ would effectively become a function of pressure in (23).

On account of these uncertainties, it was not possible to attempt a quantitative comparison between the experimental and theoretical results, nor was it practical to investigate microbubble behavior at higher insonation pressures. Marsh et al. [4] did report that increasing insonating pressure caused a downward shift in the frequency of the attenuation peak, accompanied by an increase in amplitude. This was as predicted in Figs. 6 and 7. But in order to confirm this finding, more accurate experiments would be required than could be performed with the existing apparatus. For example, an improved method for measuring the propagation speed would be required. Before such work is carried out, however, it is worthwhile considering the implications of multiple scattering for the characterization and applications of ultrasound contrast agents.

As shown above, multiple scattering would be expected to affect the attenuation and velocity of ultrasound in a microbubble suspension. The important question is whether the effects would be significant at the concentrations found in vivo. Unfortunately, the values of these concentrations are not simple to estimate. If the injected volume of contrast agent is dispersed uniformly then, assuming an average human adult blood volume of 5 liters, the average concentration will be reduced from $10^{9}$ microbubbles per milliliter to approximately $10^{5}$ microbubbles per milliliter. In this case, the effects of multiple scattering would be expected to be minimal, as demonstrated in Fig. 5. If, however, the microbubbles are not dispersed evenly, due, for example, to their being injected as a bolus, the local concentration could remain much higher, in which case multiple scattering effects might be observed. In diagnostic applications, this could limit the maximum contrast enhancement achievable with increasing dose and prevent deep structures from being imaged, owing to the rise in attenuation through the suspension, i.e., shadowing. This also could affect the percentage of microbubbles destroyed in a drug delivery application because part of the population would be effectively shielded from the incident field by the other microbubbles closer to the probe. The effect of multiple scattering upon the speed of propagation and frequency spectrum also could introduce errors into measurements of blood velocity. Further investigation is required in order to determine the magnitude of these effects.

The most significant implications of multiple scattering, however, relate to microbubble characterization experiments. The shell parameters shown in Table II were derived from measurements of the attenuation and backscat- tering coefficients from a suspension of Albunex ${ }^{\circledR}[2]$, and similar techniques have been applied to determine the properties of other agents [4], [26]. According to the results obtained above, at the concentrations used by Gorce and Schneider [26] (10 ${ }^{5}$ microbubbles per milliliter), their measurements could have been affected by multiple scattering. Hence, using a single scattering model for interpretation would have been inappropriate. This is in addition to the fact that it may not be accurate to assume linear viscoelastic behavior for all types of microbubble shell, and that the variability between individual microbubbles also will affect their response; hence, the overall attenuation coefficient. As shown in [6] the encapsulating shell is likely to be the most significant factor determining microbubble response.

Owing to the uncertainty in the variability of microbubble properties, improving the multiple scattering model as suggested in Section II would not enable more accurate microbubble characterization using acoustic techniques. It might be useful for improving the design of diagnostic procedures, for example, estimating the additional error introduced into blood velocity measurements. However, this would be the case only if the variation in the in vivo concentration could be accurately determined.

\section{Conclusions}

The results from linear modeling of ultrasound propagation in microbubble suspensions indicate that multiple scattering effects may be observed at the concentrations found in vivo if the microbubbles are of sufficient diameter and are excited at their resonance frequency. These findings are supported qualitatively by low pressure broadband attenuation measurements in suspensions of two different types of microbubbles. It was found that attenuation increased linearly with concentration in suspensions containing small, nonresonant microbubbles and nonlinearly in those containing larger, resonant microbubbles. A quantitative comparison could not be made owing to the uncertainty in the properties of the microbubble shells. The results from nonlinear modeling indicate that more significant multiple scattering effects would be expected at higher insonation pressures. In addition to a nonlinear increase in attenuation with concentration, the frequency at which attenuation was maximized was found to be higher than that predicted by single scattering models. Further work is needed to verify these results experimentally. The effects of multiple scattering should be taken into account for microbubble characterization experiments based on acoustic techniques. Accurate determination of the in vivo concentrations for microbubble suspensions is needed in order to assess the significance of multiple scattering for medical applications.

\section{Appendix A}

The linearization of (1) to obtain (2) may be achieved using the following substitutions: 


$$
R_{o 1}^{2} \ddot{x}\left(1+\left(\frac{\rho_{L}-\rho_{s}}{\rho_{s}}\right) \frac{R_{o 1}}{R_{o 2}}\right)=\frac{1}{\rho_{s}}\left(\begin{array}{c}
p_{o}(1-3 \kappa x)-p_{\infty}(t)-\frac{2 \sigma_{1}(1-x)}{R_{o 1}}-\frac{2 \sigma_{2}\left(1-x_{2}\right)}{R_{o 2}} \\
-4 \frac{(1-x)\left(1-3 x_{2}\right) \dot{x}}{R_{o 2}^{3}}\left(V_{s} \mu_{s}-R_{o 1}^{3}(1+x)^{3} \mu_{L}\right) \\
-\frac{4 V_{s} G_{s}\left(1-3 x_{2}\right)}{R_{o 2}^{3}}(1-(1+z)(1-x))-b_{\mathrm{rad}}
\end{array}\right)
$$

which simplifies to:

$$
\rho_{s} \alpha R_{o 1}^{2} \ddot{x}=\left(\begin{array}{c}
p_{o}-p_{\infty}(t)+x\left(-3 \kappa p_{o}+\frac{2 \sigma_{1}}{R_{o 1}}-\frac{2 \sigma_{2} R_{o 1}^{3}}{R_{o 2}^{4}}-\frac{4 V_{s} G_{s}}{R_{o 2}^{3}}\left(1+z\left(1+3 \frac{R_{o 1}^{3}}{R_{o 2}^{3}}\right)\right)\right) \\
-4 \frac{\dot{x}\left(V_{s} \mu_{s}-R_{o 1}^{3} \mu_{L}\right)}{R_{o 2}^{3}}-b_{\mathrm{rad}}-\frac{2 \sigma_{1}}{R_{o 1}}-\frac{2 \sigma_{2}}{R_{o 2}}+\frac{4 V_{s} G_{s} z}{R_{o 2}^{3}}
\end{array}\right)
$$

$$
\begin{aligned}
R_{1} & =R_{o 1}(1+x(t)), \\
R_{2} & =R_{o 2}\left(1+\frac{R_{o 1}^{3}}{R_{o 2}^{3}} x(t)\right), \\
R_{1 e} & =R_{o 1}(1+z), \\
\dot{R}_{1} & =R_{o 1} \dot{x}(t) \text { and } \\
\ddot{R}_{1} & =R_{o 1} \ddot{x}(t) .
\end{aligned}
$$

Applying the binomial theorem and retaining only terms linear in $x$ gives:

$$
R_{1}^{-1}=R_{o 1}^{-1}(1-x) \text { and } R_{2}^{-1}=R_{o 2}^{-1}\left(1-x_{2}\right) .
$$

Also:

$$
\frac{R_{1}}{R_{2}}=\frac{R_{o 1}(1+x)\left(1-x_{2}\right)}{R_{o 2}} \approx \frac{R_{o 1}}{R_{o 2}}
$$

because the thickness of the shell is small compared with the radius so $x-x_{2} \ll 1$.

Substituting from the above into (1) gives (A1) (see above) and finally yields the equation:

$$
\ddot{x}+\delta_{d} \dot{x}+\omega_{o}^{2} x=-\frac{p_{A} \sin (\omega t)}{\rho_{s} R_{o 1}^{2} \alpha}
$$

where $z, \delta_{d}, \omega_{o}$, and $\alpha$ are as defined previously.

\section{REFERENCES}

[1] E. Stride and N. Saffari, "Microbubble ultrasound contrast agent: A review," J. Eng. Med., vol. 217, pp. 429-447, 2003.

[2] N. de Jong, L. Hoff, T. Skotland, and N. Bom, "Absorption and scatter of encapsulated gas filled microspheres: Theoretical considerations and some measurements," Ultrasonics, vol. 30, pp. 95-103, 1992.

[3] C. Church, "The effects of an elastic solid surface layer on the radial pulsations of gas bubbles," J. Acoust. Soc. Amer., vol. 97, no. 3 , pp. 1510-1520, 1995.

[4] J. Marsh, C. Hall, M. Hughes, J. Mobley, J. Miller, and G. Brandenburger, "Broadband through-transmission signal loss measurements of Albunex ${ }^{\circledR}$ suspensions at concentrations approaching in vivo doses," J. Acoust. Soc. Amer., vol. 101, no. 2, pp. 1155-1161, 1997.

[5] Q. Chen, J. Zagzebski, T. Wilson, and T. Stiles, "Pressuredependent attenuation in ultrasound contrast agents," Ultrasound Med. Biol., vol. 8, pp. 1041-1051, 2002.
[6] E. Stride and N. Saffari, "On the destruction of microbubble ultrasound contrast agents," Ultrasound Med. Biol., vol. 29, pp. 563-573, 2003.

[7] T. Leighton, The Acoustic Bubble. London: Academic, 1994.

[8] L. Foldy, "The multiple scattering of waves. I. General theory of isotropic scattering by randomly distributed scatterers," Phys. Rev., vol. 67, pp. 107-119, 1945.

[9] F. Henyey, "Corrections to Foldy's effective medium theory for propagation in bubble clouds and other collections of very small scatterers," J. Acoust. Soc. Amer., vol. 105, no. 4, pp. 21492154, 1999.

[10] C. Feuillade, "The attenuation and dispersion of sound in water containing multiple interacting air bubbles," J. Acoust. Soc. Amer., vol. 99, pp. 3412-3430, 1996.

[11] H. Medwin, "Counting bubbles acoustically: A review," Ultrasonics, vol. 15, pp. 7-14, 1977.

[12] L. Van Wijngaarden, "On equations of motion for mixtures of liquids and gas bubbles," J. Fluid Mech., vol. 33, pp. 465-474, 1968.

[13] R. Caflisch, M. Miksis, G. Papanciolaou, and L. Ting, "Effective equations for wave propagation in bubbly liquids," J. Fluid Mech., vol. 153, pp. 259-273, 1985.

[14] K. Commander and A. Prosperetti, "Linear pressure waves in bubbly liquids: Comparison between theory and experiments," J. Acoust. Soc. Amer., vol. 85, pp. 732-746, 1989.

[15] R. Omta, "Oscillations of a cloud of bubbles of small and not so small amplitudes," J. Acoust. Soc. Amer., vol. 82, pp. 1018$1033,1987$.

[16] L. D'Agostino and C. Brennen, "Acoustical absorption and scattering cross-sections of spherical bubble clouds," J. Acoust. Soc. Amer., vol. 84, pp. 2126-2134, 1988.

[17] E. Stride and N. Saffari, "The behaviour of microbubble ultrasound contrast agent particles in whole blood," Ultrasound Med. Biol., vol. 30, pp. 144-158, 2004.

[18] J. Keller and M. Miksis, "Bubble oscillations of large amplitude," J. Acoust. Soc. Amer., vol. 68, pp. 628-633, 1980.

[19] T. Leighton, S. Meers, and P. White, "Propagation through nonlinear time dependent bubble clouds and the estimation of bubble populations from measured acoustic characteristics," Proc. R. Soc., vol. 1, p. 30, 2004.

[20] L. Collatz, Numerical Treatment of Differential Equations. 3rd ed. Berlin: Springer, 1960.

[21] W. Lord, R. Ludwig, and Z. You, "Developments in ultrasonic modelling with finite element analysis," J. Non-Destr. Eval., vol. 9, p. 129, 1990.

[22] E. Stride and N. Saffari, "The potential for thermal damage posed by microbubble ultrasound contrast agents," Ultrasonics, vol. 42, pp. 907-913, 2004.

[23] F. Duck, Physical Properties of Tissue: A Comprehensive Reference Book. London: Academic, 1990.

[24] C. MacDonald, V. Sboros, and J. Gomatam et al., "A numerical investigation of the resonance of gas-filled microbubbles: Resonance dependence on acoustic pressure amplitude," Ultrasonics, vol. 43, pp. 113-122, 2004. 
[25] M. Postema, A. Bouakaz, C. Chin, and N. de Jong, "Simulations and measurements of optical images of insonified ultrasound contrast microbubbles," IEEE Trans. Ultrason., Ferroelect., Freq. Contr., vol. 50, pp. 523-535, 2003.

[26] J. Gorce, M. Arditi, and M. Schneider, "Influence of bubble size distribution on the echogenicity of ultrasound contrast agents: A study of SonoVue," Invest. Radiol., vol. 35, pp. 661-671, 2000.

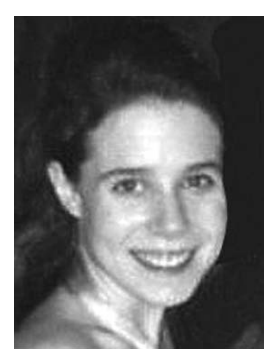

Eleanor Stride was born in London, England, in 1979. She received her bachelors degree in mechanical engineering from University College London (UCL) in 2001, graduating with first class honors and the highest marks in her year, for which she was awarded the faculty medal. She was subsequently awarded a UCL graduate studentship and, since 2002, she has been studying for her Ph.D. degree on the characterization and design of ultrasound contrast agents, which she has recently completed.

She is currently working as a member of the Ultrasonics Group at UCL, where she is continuing her research on ultrasound contrast agents and where she also lectures in mathematics. She is an associate member of the Institute of Physics (IoP) and also sits on the IoP Physical Acoustics Group Committee. In addition to ultrasound contrast agents, her interests include reconstruction algorithms for ultrasonic tomography.

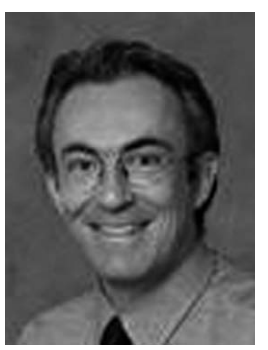

Nader Saffari has a B.Sc. degree in electrical and electronic engineering from Leeds University, UK, a M.Sc. degree in microwaves and modern optics from University College London (UCL), and a Ph.D. degree, awarded in 1986, again from UCL. His dissertation was on the characterization of surface defects using ultrasound and was a combination of numerical model development and experimental studies. After completing his studies, he worked for 6 years as a consultant in the field of ultrasonic nondestructive evaluation for clients in the aerospace and defense sectors.

Since 1992 he has been with the Department of Mechanical Engineering, UCL, where he is a Reader in Ultrasonics and heads the Ultrasonics Group. His current research interests include ultrasonic characterization of advanced materials such as aerospace composites and biomaterials, acoustical imaging, signal and image processing in acoustics, mathematical modeling of ultrasound propagation and scattering in inhomogeneous materials, and design of ultrasound contrast agents for medical diagnostic and therapeutic applications. Within his research group he has developed both a strong theoretical modeling activity and extensive experimental capabilities. 